PROCEEDINGS OF THE

AMERICAN MATHEMATICAL SOCIETY

Volume 127, Number 4, April 1999, Pages 973-976

S 0002-9939(99)04643-2

\title{
ASSOCIATIVE RINGS SATISFYING THE ENGEL CONDITION
}

\author{
D. M. RILEY AND MARK C. WILSON \\ (Communicated by Ken Goodearl)
}

\begin{abstract}
Let $C$ be a commutative ring, and let $R$ be an associative $C$ algebra generated by elements $\left\{x_{1}, \ldots, x_{d}\right\}$. We show that if $R$ satisfies the Engel condition of degree $n$, then $R$ is upper Lie nilpotent of class bounded by a function that depends only on $d$ and $n$. We deduce that the Engel condition in an arbitrary associative ring is inherited by its group of units, and implies a semigroup identity.
\end{abstract}

As part of his positive solution of the restricted Burnside problem ([5]), Zel'manov proved that every finitely generated Lie ring satisfying the Engel condition

$$
[x, \underbrace{y, y, \ldots, y}_{n}]=0
$$

is nilpotent. In Lie algebras over a field of characteristic zero, the Engel condition implies nilpotence - not just local nilpotence. This result was proved by Kemer ([3]) for the class of Lie algebras arising from associative algebras, and later by Zel'manov ([4]) for general abstract Lie algebras. It is known, however, that this result fails to hold for general Lie rings, and in particular fails for Lie algebras arising from associative algebras over fields of positive characteristic ([7],[10]). Perhaps the simplest example is the tensor square of the (nonunital) Grassmann algebra of a countably-infinite-dimensional vector space over a field of characteristic $p>2$. In fact, it is nil of bounded index $2 p$ but not Lie nilpotent; which is to say, it is not nilpotent when considered as a Lie algebra $([8],[9])$.

Let $C$ be a commutative (unital) ring, and let $R$ be a finitely generated (associative) $C$-algebra. It is not strictly necessary for us to assume that $R$ is unital, but we shall as a matter of convenience. The primary purpose of this note is to prove that if $R$ satisfies the Engel condition, then $R$ is Lie nilpotent. Since such rings need not be finitely generated as Lie rings, our result is not included in Zel'manov's. Shalev, however, proved the above result in the case when $C$ is a field of positive characteristic ([12]).

Furthermore, we are able to show that $R$ is Lie nilpotent in a stronger sense. Define a descending central series of associative ideals $\left\{\gamma^{i}(R)\right\}$ in $R$ by $\gamma^{1}(R)=$ $R, \gamma^{i+1}(R)=\left\langle\left[\gamma^{i}(R), R\right]\right\rangle=\left[\gamma^{i}(R), R\right] R$. Then $R$ is upper Lie nilpotent of class $s$

Received by the editors March 20, 1997 and, in revised form, April 15, 1997 and July 29, 1997. 1991 Mathematics Subject Classification. Primary 16R40; Secondary 16W10, 17B60, 16 U60.

Key words and phrases. Engel identity, Lie nilpotent, strongly Lie nilpotent, upper Lie nilpotent, nonmatrix.

The first author received support from NSF-EPSCoR in Alabama and the University of Alabama Research Advisory Committee.

The second author was supported by a NZST Postdoctoral Fellowship. 
if $\gamma^{s}(R) \neq 0$ and $\gamma^{s+1}(R)=0$. Although upper Lie nilpotence clearly implies Lie nilpotence, the converse need not be true (as was conjectured by S.A. Jennings). Indeed, the Grassmann algebra over a field of characteristic not 2 is Lie nilpotent of class 2 but not upper Lie nilpotent, while Gupta and Levin constructed a similar example in characteristic $2([2])$.

Theorem. Let $C$ be a commutative ring, and let $R$ be a finitely generated associative $C$-algebra which is generated by d elements and satisfies the Engel condition of degree $n$. Then $R$ is upper Lie nilpotent, of class bounded by a function depending on $d$ and $n$ only.

Corollary 1. Let $R$ be an associative ring. If $R$ satisfies the Engel condition of degree $n$, then its group of units $\mathcal{U}(R)$ satisfies the Engel condition of degree $m$ for some $m$ depending only on $n$.

Corollary 2. Let $R$ be an associative ring. If $R$ satisfies the Engel condition, then $R$ satisfies a semigroup identity.

A semigroup identity is the equality of two words in the free monoid on countably many letters. We remark that Corollary 2 settles the ring-theoretic analogue of a problem raised by Shirshov ([6, Problem 2.82]): does the variety of groups satisfying an Engel identity satisfy a semigroup identity?

\section{Proofs}

All associative rings considered below will be assumed to be unital. We denote the usual descending central series of a group or Lie ring by $\left\{\gamma_{i}(\cdot)\right\}$.

Proposition 1. Suppose that $R$ is generated as an associative $C$-algebra by a Lie subring $L$ which is nilpotent of class $s$. If $\gamma_{2}(L)$ is associative nilpotent of index $t+1$, then $R$ is upper Lie nilpotent of class at most st +1 .

Proof. We first collect a few simple facts that follow from the identities

$$
[x, y z]=y[x, z]+[x, y] z, \quad[x y, z]=x[y, z]+[x, z] y .
$$

Notice that the Lie ring $L$ acts on $R$ via the ad map. If a subset $X$ in $R$ is stable under this action, then $X R=R X$. Also, $[X, R] \subseteq[X, L] R$. In particular, $\gamma_{i}(L) R=R \gamma_{i}(L)$ and $\left[\gamma_{i}(L), R\right] \subseteq \gamma_{i+1}(L) R$ for each $i$. It follows that

$$
\gamma^{2}(R)=\gamma_{2}(L) R
$$

and

$$
\left[\gamma_{i}(L) R, R\right] \subseteq \gamma_{i}(L) \gamma_{2}(L) R+\gamma_{i+1}(L) R .
$$

Put $I_{i}=\gamma_{i}(L) R$ for each $i$. Then $I_{i}$ is an associative ideal of $R$ with the property that

$$
\left[I_{i}, R\right] \subseteq I_{i} I_{2}+I_{i+1}
$$

We claim that, for all $n \geq 2$,

$$
\gamma^{n}(R) \subseteq \sum I_{n_{1}} \cdots I_{n_{k}}
$$

where the sum is over all $\left(n_{1}, \ldots, n_{k}\right)$ with $n_{i} \geq 2$ and $\sum_{i}\left(n_{i}-1\right)=n-1$. 
The claim is true for $n=2$ since $\gamma^{2}(R)=I_{2}$. If $n>2$, then the claim follows by induction and the observation

$$
\begin{aligned}
{\left[I_{n_{1}} \cdots I_{n_{k}}, R\right] } & \subseteq \sum_{i=1}^{k} I_{n_{1}} \cdots I_{n_{i-1}}\left[I_{n_{i}}, R\right] I_{n_{i+1}} \cdots I_{n_{k}} \\
& \subseteq \sum_{i=1}^{k} I_{n_{1}} \cdots I_{n_{i-1}}\left(I_{n_{i}} I_{2}+I_{n_{i}+1}\right) I_{n_{i+1}} \cdots I_{n_{k}} .
\end{aligned}
$$

If all $n_{i} \leq s$ and $k \leq t$, then $n-1 \leq s t$. Hence if $n>1+s t$, then each monomial above is zero since $I_{n_{i}} \subseteq I_{2}$ and $I_{2}^{t+1}=I_{s+1}=0$.

Proposition 2. Let $n$ and $d$ be positive integers. Then $F(d, n)$, the relatively free $d$-generated ring satisfying the Engel condition of index $n$, is upper Lie nilpotent.

Proof. We shall employ some well-known results from PI-theory, for which [11] is a sufficient reference. Write $R=F(d, n)$.

Let $J$ be the Jacobson radical of $R$. Because $R$ is a finitely generated PI-ring, $J$ is nilpotent by a theorem of Razmyslov. We claim that $\gamma^{2}(R) \subseteq J$; which is to say, $R / J$ is commutative. Indeed, $R / J$ is a semiprimitive PI-ring. Thus by a theorem of Kaplansky, it is a subdirect product of full matrix rings over division rings, $M_{n}(D)$ say, where $D$ is finite dimensional over its centre $F$. As $M_{2}(F)$ does not satisfy the Engel condition, we must have $M_{n}(D)=D$. If $F$ is finite, then $D=F$, whereas if $F$ is infinite, then $D$ has precisely the same identities as $M_{m}(F)$ for some $m \geq 1$. But again, the latter possibility can only occur if $m=1$, and so either way $D=F$. Thus, $R / J$ is a subdirect product of fields, and therefore commutative. It follows that $\gamma^{2}(R)$ is nilpotent.

Let $L$ be the Lie subring of $R$ generated by the $d$-many associative generators of $R$. Since $\gamma^{2}(R)$ is nilpotent, $R$ is (associatively nilpotent)-by-commutative, and hence Lie solvable. Thus $L$ is a finitely generated solvable engelian Lie ring, which is nilpotent by a theorem of Gruenberg ([1]). The result now follows directly from Proposition 1.

We denote the upper nilpotence class in the conclusion of Proposition 2 by $f(d, n)$.

To prove the theorem, let $S$ be the subring of $R$ generated by its $d C$-generators. Then $S$ is upper Lie nilpotent of class $f(d, n)$ since $S$ is a homomorphic image of $F(d, n)$. Since upper Lie nilpotence corresponds to a multilinear identity and $R$ is a homomorphic image of $S \otimes_{\mathbb{Z}} C$, the result now follows from Proposition 2.

We now prove Corollary 1 . Let $R$ be a ring satisfying the Engel condition of degree $n$, and let $S$ be the subring in $R$ generated by $\left\{u, v, u^{-1}, v^{-1}\right\}$, where $u, v$ are arbitrary units in $R$. Then $S$ is upper Lie nilpotent of class at most $f(4, n)$. The result now follows from the inclusion

$$
\gamma_{m}(\mathcal{U}(S)) \subseteq 1+\gamma^{m}(S)
$$

which is readily established by induction.

Finally, we prove Corollary 2. Suppose that $R$ satisfies the Engel condition of degree $n$, and let $S$ be an arbitrary 2-generated subring of $R$. Then $S$ is Lie nilpotent of class at most $c=f(2, n)$. It follows by induction that $S$ satisfies the Morse identity $x_{c}=y_{c}$ defined by $x_{1}=x y, y_{1}=y x, x_{i+1}=x_{i} y_{i}, y_{i+1}=y_{i} x_{i}$. Hence $R$ also satisfies the semigroup identity $x_{c}=y_{c}$. 


\section{REFERENCES}

[1] K. W. Gruenberg, Two theorems on Engel groups, Proc. Cambridge Philos. Soc. 49 (1953), 377-380. MR 14:1060f

[2] N. D. Gupta and F. Levin, On the Lie ideals of a ring, J. Algebra 81 (1983), 225-231. MR 84i: 16036

[3] A. Kemer, Non-matrix varieties, Algebra and Logic 19 (1981), 157-178.

[4] E. I. Zel'manov, Engelian Lie algebras, Siberian Math. J. 29 (1988), 777-781.

[5] _. On the restricted Burnside problem, Siberian Math. J. 30 (1990), 885-891. MR 92a:20041

[6] V. D. Mazurov and E. I. Khukhro (eds.), Unsolved problems in group theory. The Kourovka notebook., 13th ed., Russian Academy of Sciences Siberian Division, Institute of Mathematics, Novosibirsk, 1995. MR 97d:20001

[7] Yu. P. Razmyslov, On Lie algebras satisfying the Engel condition, Algebra and Logic 10 (1971), 21-29.

[8] D. M. Riley, The tensor product of Lie soluble algebras, Arch. Math. (Basel) 66 (1996), 372-377. MR 97e: 16057

[9] D. M. Riley and V. Tasić, The transfer of a commutator law from a nil-ring to its adjoint group, Canad. Math. Bull. 40 (1997), 103-107. CMP 97:11

[10] E. Rips and A. Shalev, The Baer condition for group algebras, J. Algebra 140 (1991), 83-100. MR 92g: 17008

[11] L. Rowen, Ring theory, Academic Press, 1988. MR 89h:16001

[12] A. Shalev, On associative algebras satisfying the Engel condition, Israel J. Math. 67 (1989), 287-290. MR 91c:16025

Department of Mathematics, University of Alabama, Tuscaloosa, Alabama 35487 0350

E-mail address: driley@gp.as.ua.edu

Department of Mathematics, University of Auckland, Private Bag 92019 Auckland, New ZeALAND

E-mail address: wilson@math.auckland.ac.nz

Current address: Department of Mathematics, Statistics and Computer Science, University of Illinois at Chicago, Chicago, Illinois 60607-7045 